\title{
Editorial
}

\section{Ischaemia, action potentials, and refractoriness}

The study by Sutton and colleagues in this issue is the first to measure in humans the changes in the left ventricular refractory period and the duration of the ventricular monophasic action potential during the first minutes of acute ischaemia. ${ }^{1}$ As the authors mention, the experimental data on this subject are rather confusing. Most studies report on a shortening of the refractory period, but lengthening, shortening followed by lengthening, and lengthening followed by shortening have been reported as well. The classical method to determine refractory period duration is to pace the heart with a train of regular stimuli, and to introduce after every eighth to 10th basic stimulus a premature stimulus that is stronger than the basic stimulus. The shortest coupling interval of the premature stimulus that results in a propagated response is then defined as the refractory period at the site of stimulation. The discrepancies between the various experimental studies can be attributed to the use of different current strengths of the premature stimulus (usually 2-4 times diastolic threshold, but intensities of 10-15 times diastolic threshold have been used as well ${ }^{2}$ ), and to the type of ischaemia-global or regional.

During ischaemia the diastolic threshold for excitation increases several fold; therefore, the intensity of the premature stimuli necessary to elicit a response is usually continuously increased during the development of ischaemia. In experimental models of regional ischaemia, the use of strong currents may lead to excitation of tissue at a considerable distance from the stimulating electrode. ${ }^{3}$ In the "lateral border zone", where ischaemic cells are exposed to much lower extracellular potassium $\left(\mathrm{K}^{+}\right)$concentrations than in the "central ischaemic zone", current requirements may even be less than in normal myocardium. ${ }^{4}$ Because in this lateral border zone, action potentials are shorter than those in normal myocardium, ${ }^{5}$ high currents delivered to the central ischaemic zone may excite tissue in the lateral border at short coupling intervals, and one may erroneously conclude that refractory periods during ischaemia shorten. Since action potentials in the central ischaemic zone shorten as well, the conclusion may be drawn that the normal relation between action potential duration and refractory period is maintained during ischaemia. Experimental studies, in which microelectrode recordings were made as close as possible to the stimulating electrode, have shown that during ischaemia this relation is lost and that so-called post-repolarisation refractoriness develops, where recovery of excitability lags behind full repolarisation. ${ }^{36}$ This is confirmed in the study by Sutton and colleagues. ${ }^{1}$ The beauty of their protocol is that during coronary bypass surgery a brief, three minute period of ischaemia followed by reperfusion preconditions the heart and protects it during the subsequent long ischaemic period used for graft anastomoses. The electrophysiologic measurements made during the three minute preconditioning ischaemic period therefore inflict no harm on the patient.

\section{Differences between findings in human hearts and experimental studies}

There are some important differences between the findings in the human hearts with coronary artery disease in the present study and earlier experimental studies on normal canine or porcine hearts with acute coronary artery occlusion. In the first place, the changes in action potential duration and refractoriness develop much more rapidly in the human hearts. The most important factors that determine these changes are the loss of $\mathrm{K}^{+}$ions from ischaemic cells and the consequent accumulation of $\mathrm{K}^{+}$ions in the extracellular space, combined with severe hypoxia. ${ }^{5}$ It would be of interest to determine the rise in extracellular $\mathrm{K}^{+}$in the hearts of the patients, and in case it is faster than in experimental models, to find out the reasons. Is there already a malfunctioning $\mathrm{Na}^{+} / \mathrm{K}^{+}$pump in these hearts? Is there already a change in function of the ATP sensitive $\mathrm{K}^{+}$ channel, or a faster loss of intracellular ATP? Is there a greater cellular loss of anions?

Secondly, although of minor importance, it has been shown that very early during the development of ischaemia, action potential duration transiently lengthens owing to a drop in temperature in the ischaemic myocardium, followed by shortening, largely caused by the rise in extracellular $\mathrm{K}^{+2}$. It would be of interest to know what the temperature of the globally ischaemic hearts of the patients was.

Finally, an intriguing result was obtained by using two groups: group, 1 in which the premature stimuli had a strength of four times diastolic, pre-ischaemic threshold; and group 2, where the intensity was only twice diastolic threshold. In both groups the duration of the monophasic action potential decreased by an average of $67 \mathrm{~ms}$. However, in group 1, the refractory period lengthened by $14 \mathrm{~ms}$ on average, and in group 2 it lengthened by $92 \mathrm{~ms}$. This is probably caused by an increase in diastolic threshold during ischaemia, so that the premature stimulus was no longer twice diastolic threshold, but much less. This emphasises the importance of the current strength used to determine refractory periods.

In summary, this first study in diseased human hearts, impeccably performed, highlights the differences in changes in repolarisation and refractoriness during acute ischaemia, and underscores the difficulties in extrapolating findings in experimental models to the human heart with coronary artery disease.

Cardiovascular Research,

MICHIEL J JANSE

Academic Medical Center, Room f1-27

Meibergdreef 9 ,

1105 AZ Amsterdam,

The Netherlands

m.j.janse@amc.uva.nl

1 Sutton PMI, Taggart P, Opthof T, et al. Repolarisation and refractoriness during early ischaemia in humans. Heart 2000;84:365-9.

2 Wit AL, Janse MJ. The ventricular arrhythmias of ischemia and infarction. Electrophysiologic mechanisms. Mount Kisco, New York: Futura Publishing Electrophysiologic

3 Janse MJ, Capucci A, Coronel R, et al. Variability of recovery of excitability in the normal canine and ischaemic porcine heart. Eur Heart $\mathcal{f}$ $1985 ; 6$ (suppl D):41-52. 
4 Coronel R, Wilms-Schopman FJG, Opthof T, et al. Injury currents and gradients of diastolic threshold, TQ potential, and extracellular potassium concentration during acute regional ischemia in the isolated perfused pig
heart. Circ Res 1991;68:1241-9.

5 Kodama I, Wilde AAM, Janse MJ, et al. Combined effects of hypoxia, hyper- kalemia and acidosis on membrane action potentials and excitability of guinea pig ventricular muscle. $7 \mathrm{Mol}$ Cell Cardiol 1984;16:246-59.

6 Downar E, Janse MJ, Durrer D. The effect of acute coronary artery occlusion on subepicardial transmembrane potentials in the intact porcine heart. Circulation 1977;56:217-24.

\section{IMAGES IN CARDIOLOGY}

\section{A giant aneurysm in coronary-pulmonary artery fistula associated with mural thrombus}

A 66 year old man was referred for further cardiac examination regarding an abnormal finding at $x$ ray (top left) that had gradually enlarged over a 20 year period. At his local hospital a coronary angiogram had revealed a large aneurysm in a coronary pulmonary artery fistula. The fistula originated from the proximal left anterior descending artery, and in the mid portion there was a giant aneurysm measuring $27 \mathrm{~mm}$ (long axis) $\times 20 \mathrm{~mm}$ (short axis) filled with contrast material (top right). However, it was difficult to determine from the angiogram the connecting site to the pulmonary artery because of retention of the contrast material in the aneurysm. Echocardiography revealed a thick mural thrombus within the aneurysm (bottom left, arrows) (An, aneurysm; PA, pulmonary artery); the inner dimension was $22 \mathrm{~mm} \times 24 \mathrm{~mm}$, which was compat-
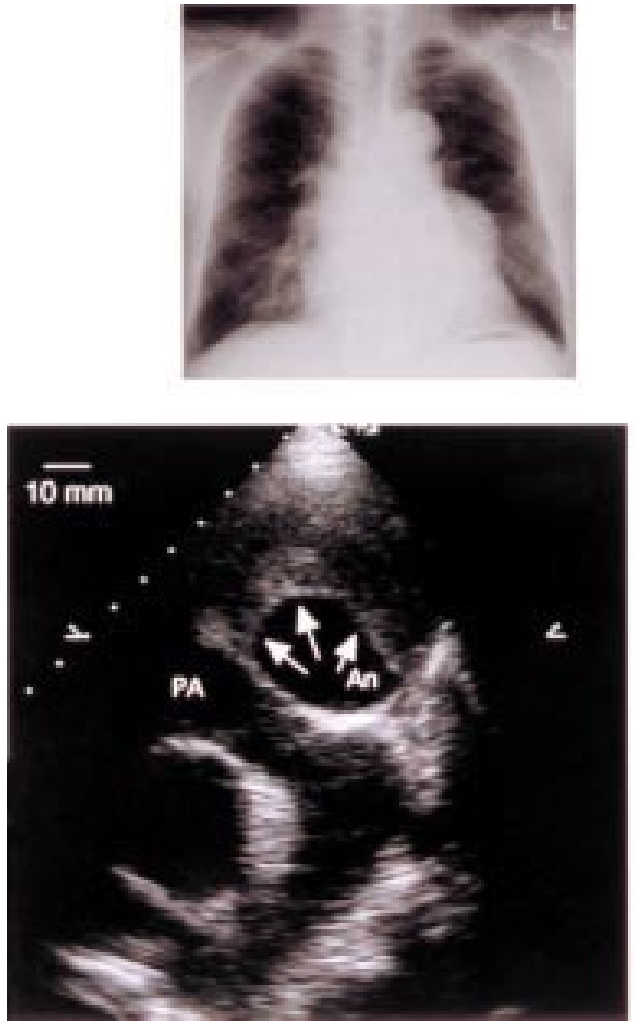

tible with the short axis dimension determined by angiography. However, the outer dimension of the aneurysm as determined by echocardiography was much greater than the dimensions determined by angiography, which explains the huge abnormal shadow in the plain chest $x$ ray. Colour Doppler imaging clearly demonstrated flow from the fistula to the pulmonary artery through the aneurysm (bottom right). The patient underwent resection of the aneurysm with reconstruction of the coronary artery. In this case echocardiography accurately determined the morphology of the aneurysm in terms of its size and site of connection to the pulmonary artery.

MASAKAZU YAMAGISHI YOSHIO YASUMURA KO BANDO
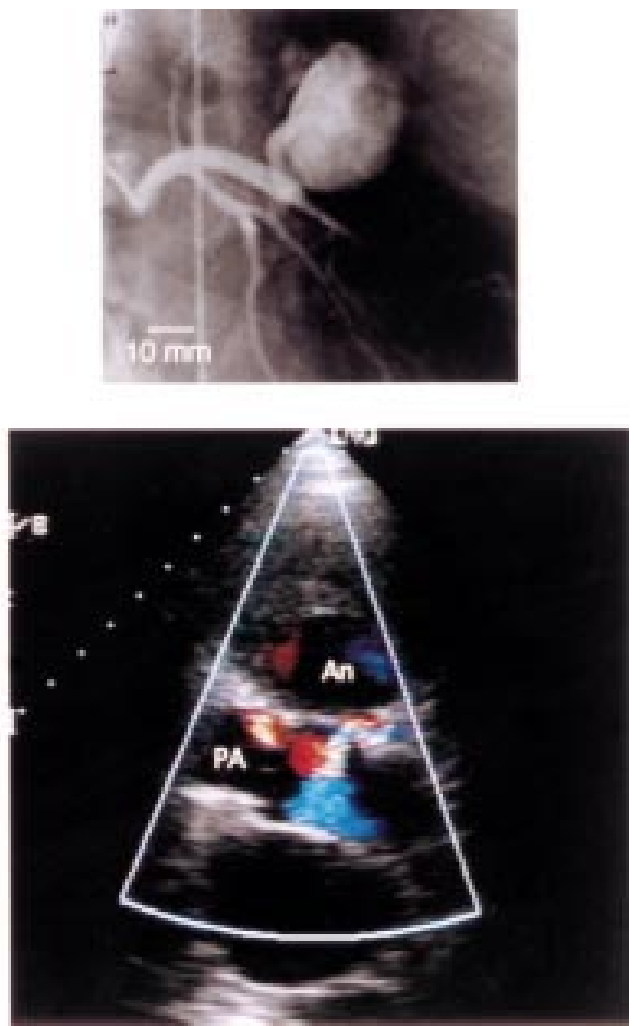